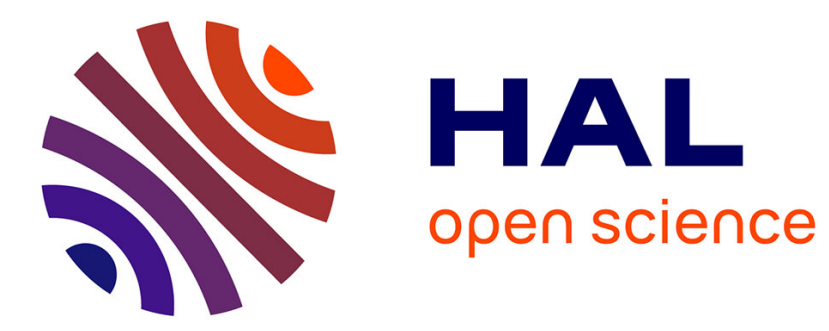

\title{
Variability and Confidence Intervals of the Power Measured in a Reverberation Chamber
}

Florian Monsef, Andrea Cozza

\section{To cite this version:}

Florian Monsef, Andrea Cozza. Variability and Confidence Intervals of the Power Measured in a Reverberation Chamber. IEEE Transactions on Electromagnetic Compatibility, 2014, 56 (5), pp.12381241. 10.1109/TEMC.2014.2305845 . hal-01066485

\section{HAL Id: hal-01066485 \\ https://hal-centralesupelec.archives-ouvertes.fr/hal-01066485}

Submitted on 20 Sep 2014

HAL is a multi-disciplinary open access archive for the deposit and dissemination of scientific research documents, whether they are published or not. The documents may come from teaching and research institutions in France or abroad, or from public or private research centers.
L'archive ouverte pluridisciplinaire HAL, est destinée au dépôt et à la diffusion de documents scientifiques de niveau recherche, publiés ou non, émanant des établissements d'enseignement et de recherche français ou étrangers, des laboratoires publics ou privés. 


\title{
Variability and confidence intervals of the
}

\section{power measured in a reverberation chamber}

\author{
Florian Monsef, Member IEEE, Andrea Cozza, Senior Member IEEE
}

\begin{abstract}
The relative variance (or variability) of the average power measured in a reverberation chamber is derived. It is found to be a function of the average number of modes overlapping in the average modal bandwidth found at the working frequency. The model can predict the average-power variance from under- to over-moded regimes. Good agreement with experimental and simulation data is obtained. Confidence intervals of the estimate of the average-power variability are computed for different chamber regimes and a varying number of independent stirrer positions.

Index Terms-reverberation chamber (RC), electromagnetic compatibility (EMC), modal analysis, parametric statistics, cavity resonators.
\end{abstract}

\section{INTRODUCTION}

For immunity tests, one expects that over a full stirrer turn, peak values of the field are found to uniformly stress a device under test (DUT). The ability to estimate this uniformity level is essential to quantify the magnitude of the stress put onto a DUT [1] [2] [3].

However, the use of maximum values give access to a reduced number of samples from which ensues an important dispersion. This fact is pointed out in [2] which explains the benefit of using average values of the power received on a reference to estimate peak values.

Florian Monsef and Andrea Cozza are with the Départment de Recherche en Electromagnétisme, Laboratoire des Signaux et Systèmes, UMR8506, Univ Paris-Sud, SUPELEC, CNRS, 91190 Gif-sur-Yvette, France.
The interest in using the average power has also recently been presented in [4], in which the susceptibility level was estimated by using the probability of failure of a DUT.

Although the methods mentioned above assume an overmoded regime, in practice, the use of ModeStirred Reverberation Chambers (MSRCs) is not restricted to that case. So, the derivation, in any regime, of the variability of the average power received on a reference antenna is useful, not only for possible extensions of the work dealing with the maximum stress estimation, but also for wanted and/or unwanted emissions tests for which average values are the quantity of interest [1].

To this end we refer to a recent work [5] which aims at deriving the variability of the electric-energy density. Unlike in [5], we present an approach that does not make use of the statistical-bandwidth concept. After a brief summary of the field model in Section II, we will proceed to the derivation of the power variability in Section III. Finally, in Section IV we present the model validation using experimental and simulations results. Confidence intervals (CI) (at the 95\% confidence level) are of practical use and are also computed.

\section{FIELD MODEL}

For the specific case of an MSRC, the electric field, referred herein to as $\mathbf{E}\left(\mathbf{r}, f_{w}\right)$, at a position $\mathbf{r}$ and at a working frequency $f_{w}$, on the basis of a 
modal approach has been presented for the first time in [6]. The approach describing the modal parameters as independent random variables has been presented more recently in [7] and used in [8] and [5]. The variability of the electric-energy density has been derived in the aforementioned works by introducing, for the latter, the statistical bandwidth concept.

Following the approach presented in [7] and [5], the electric-field model is,

$$
\mathbf{E}\left(\mathbf{r}, f_{w}\right)=\sum_{i=1}^{\infty} \tilde{\gamma}_{i} \psi_{i}\left(f_{w}\right) \hat{\xi}_{i}(\mathbf{r})
$$

where the complex quantities $\tilde{\gamma}_{i}$ are regarded as equivalent modal weights, whose real and imaginary parts follow a normal law, $\hat{\xi}_{i}(\mathbf{r})$ is a unitary polarization vector related to the ith mode, which is assumed to be uniformly distributed over $4 \pi \mathrm{sr}$, and $\psi_{i}(f)$ refers to the modal frequency response which is of Lorentzian shape. For further details the reader can refer to [7] and [5].

\section{DERIVATION OF THE RELATIVE VARIANCE}

We consider the electric power $P_{r}$ measured on a reference antenna. Let us introduce the relative variance of $P_{r}$, referred to as $\varsigma_{P}^{2}$, and defined as,

$$
\varsigma_{P}^{2}=\frac{\mathrm{E}\left[P_{r}^{2}\right]}{\mathrm{E}^{2}\left[P_{r}\right]}-1
$$

where $\mathrm{E}[\cdot]$ refers to the ensemble average operator.

A general expression of the power received by an antenna in an MSRC can be found in [ [9], pp. 14]. If we consider the classic case of an electrically small linearly polarized antenna, the power received can be expressed as,

$$
P_{r}\left(\mathbf{r}, f_{w}\right)=C\left|\mathbf{E}\left(\mathbf{r}, f_{w}\right) \cdot \hat{\xi}_{\hat{a}}\right|^{2}
$$

where $\hat{\xi}_{\hat{a}}$ is the antenna polarization unit vector and the constant $C=A_{\text {eff }} / \eta_{0} ; \eta_{0}=377 \Omega$ being the wave impedance of air and $A_{e f f}$ the antenna's effective area.
Using (1), $P_{r}\left(\mathbf{r}, f_{w}\right)$ can be recast as follows,

$$
\begin{aligned}
P_{r}\left(\mathbf{r}, f_{w}\right) & =C \sum_{i=1}^{\infty}\left|\tilde{\gamma}_{i}\right|^{2}\left|\psi_{i}\left(f_{w}\right)\right|^{2}\left|u_{i}\right|^{2} \\
& +\sum_{i \neq j}^{\infty} \tilde{\gamma}_{i} \tilde{\gamma}_{j}^{*} \psi_{i}\left(f_{w}\right) \psi_{j}^{*}\left(f_{w}\right) u_{i} u_{j}^{*},
\end{aligned}
$$

where $u_{i}=\hat{\xi}_{i} \cdot \hat{\xi}_{\hat{a}}^{*}$.

Modal polarization vectors $\hat{\xi}_{i}$ being assumed to be uniformly distributed over $4 \pi \mathrm{sr}$, we can show that the scalar $u_{i}$ is uniformly distributed over $[-1:+1]$.

To compute the average power received over an ensemble of stirrer positions, the operator $\mathrm{E}_{\alpha}[$.$] , defined$ as the expected-value operator applied to the random variable $\alpha$, is used, and the following notations are adopted,

$$
\mu_{n}=\mathrm{E}_{\tilde{\gamma}_{\mathrm{i}}}\left[\left|\tilde{\gamma}_{i}\right|^{n}\right], \kappa_{n}=\mathrm{E}_{\mathrm{u}_{\mathrm{i} \hat{\mathrm{r}}}}\left[\left|u_{i \hat{r}}\right|^{n}\right]
$$

The first step consists in computing the expected mean value of $P_{r}\left(\mathbf{r}, f_{w}\right)$, referred to as $\mu_{P_{r}}$, which reads,

$$
\begin{aligned}
& \mu_{P_{r}}=\mathrm{E}\left[P_{r}\left(\mathbf{r}, f_{w}\right)\right] \\
& =C \mathrm{E}_{\tilde{\gamma}_{i}, f_{i}, u_{i \hat{r}}}\left[\sum_{i=1}^{\infty}\left|\tilde{\gamma}_{i}\right|^{2}\left|\psi_{i}\left(f_{w}\right)\right|^{2}\left|u_{i \hat{r}}\right|^{2}\right. \\
& \left.\quad+\sum_{i \neq j}^{\infty} \tilde{\gamma}_{i} \tilde{\gamma}_{j}^{*} \psi_{i}\left(f_{w}\right) \psi_{j}^{*}\left(f_{w}\right) u_{i \hat{r}} u_{j \hat{r}}^{*}\right]
\end{aligned}
$$

Recalling, on the one hand, the assumption of independence between modal weights [7], resonance frequencies and the $\left\{u_{i}\right\} \mathrm{s}$, and, on the other hand, that $\tilde{\gamma}_{i}$ are centered random variables, (6) can be recast as follows,

$\mu_{P_{r}}=C \mathrm{E}_{f_{i}}\left[\sum_{i=1}^{\infty} \mathrm{E}_{\tilde{\gamma}_{i}}\left[\left|\tilde{\gamma}_{i}\right|^{2}\right]\left|\psi_{i}\left(f_{w}\right)\right|^{2} \mathrm{E}_{u_{i \hat{r}}}\left[\left|u_{i \hat{r}}\right|^{2}\right]\right]$.

Using the notations given by $(5), \mu_{P_{r}}$ reads,

$$
\mu_{P_{r}}=C \frac{\mu_{2} \kappa_{2}}{\eta_{0}} \mathrm{E}_{f_{i}}\left[\sum_{i=1}^{\infty}\left|\psi_{i}\left(f_{w}\right)\right|^{2}\right] .
$$

In order to compute (8), special care must be taken with the derivation of the expected-value term applied to the ensemble of the resonance frequencies. The key property lies in the assumption of that the modal bandwidth $f_{i} / \bar{Q}\left(f_{w}\right)$ is sufficiently constant. This allows us 
to replace the $\psi_{i}(f)$ by a frequency template referred to as $\psi_{0}(f)$ such that : $\psi_{i}(f)=\psi_{0}\left(f-f_{w}\right)$ [5].

The sum, in the inner part of $\mathrm{E}_{f_{i}}[$.$] , includes a$ set of eigenfrequencies related, in practice, to a given stirrer position. For different stirrer positions, each of these eigenfrequencies is assumed to be uniformly distributed within the mean spacing between adjacent modes, here $\Delta f$.

The expected-value term applied to the ensemble of the resonance frequencies can then be expressed as,

$$
\mathrm{E}_{f_{i}}\left[\sum_{i=1}^{\infty}\left|\psi_{i}\left(f_{w}\right)\right|^{2}\right]=\int_{0}^{\infty} \frac{1}{\Delta f}\left|\psi_{i}(f)\right|^{2} \mathrm{~d} f_{i} .
$$

Using the approximation stated previously, it follows that,

$$
\mathrm{E}_{f_{i}}\left[\sum_{i=1}^{\infty}\left|\psi_{i}(f)\right|^{2}\right]=\int_{0}^{\infty} \frac{1}{\Delta f}\left|\psi_{0}\left(f-f_{w}\right)\right|^{2} \mathrm{~d} f .
$$

The mean spacing between adjacent eigenfrequencies corresponds to the inverse of the modal density and is therefore a function of frequency. However, the sharpness of the resonances is such that the mean spacing that intervenes significantly is the one "sampled" at the working frequency $f_{w}[5] ; \Delta f$ can then conveniently be taken out of the integral. Hence,

$$
\mathrm{E}_{f_{i}}\left[\sum_{i=1}^{\infty}\left|\psi_{i}\left(f_{w}\right)\right|^{2}\right] \simeq \frac{m\left(f_{w}\right) \pi}{2 B_{M}},
$$

where $B_{M}$ is the $-3-\mathrm{dB}$ modal bandwidth, leading finally to,

$$
\mu_{P_{r}}=C \frac{\mu_{2} \kappa_{2}}{\eta_{0}} \frac{m\left(f_{w}\right) \pi}{2 B_{M}},
$$

where $m\left(f_{w}\right)$ is the modal density at working frequency.

To establish the relative variance of the power given by (2), we need to derive the $\mathrm{E}\left[P_{r}^{2}\left(\mathbf{r}, f_{w}\right)\right]$ term which can be expressed as follows,

$$
\begin{aligned}
\mathrm{E}\left[P_{r}^{2}\right]=C^{2} & \left(\mu_{4} \kappa_{4} \mathrm{E}_{f_{i}}\left[\sum_{i=1}^{\infty}\left|\psi_{i}\left(f_{w}\right)\right|^{4}\right]\right. \\
& \left.+2 \mu_{2}^{2} \kappa_{2}^{2} \mathrm{E}_{f_{i}}\left[\sum_{i \neq j}^{\infty}\left|\psi_{i}\left(f_{w}\right)\right|^{2}\left|\psi_{j}\left(f_{w}\right)\right|^{2}\right]\right),
\end{aligned}
$$

where [5],

$$
\begin{aligned}
& \mathrm{E}_{f_{i}}\left[\sum_{i=1}^{\infty}\left|\psi_{i}\left(f_{w}\right)\right|^{4}\right] \simeq \frac{m\left(f_{w}\right) \pi}{4 B_{M}^{3}} \\
& \mathrm{E}_{f_{i}}\left[\sum_{i \neq j}^{\infty}\left|\psi_{i}\left(f_{w}\right)\right|^{2}\left|\psi_{j}\left(f_{w}\right)\right|^{2}\right] \simeq \frac{m\left(f_{w}\right) \pi}{4 B_{M}^{3}}\left[\pi m\left(f_{w}\right) B_{M}-1\right] .
\end{aligned}
$$

Moreover, recalling the statistical distributions assumed over $\gamma_{i}$ and $u_{i, \hat{r}}$, we have $\mu_{4} / \mu_{2}^{2}=2$ and $\kappa_{4} / \kappa_{2}^{2}=9 / 5$; it follows that $\varsigma_{P}^{2}$ reads,

$$
\varsigma_{P}^{2}=1+\frac{8}{5 \pi M_{M}} .
$$

As observed for the electric energy density in [7], the variability of the power is also a decreasing function with the number $M_{M}$ defined as being the number of modes overlapping in the average -3-dB-modal bandwidth.

For an infinite number of modes, i.e. for $M_{M} \mapsto$ $\infty$, the special case of the overmoded regime is found since (15) reduces to one, which equals the relative variance of the well-known $\chi_{2}^{2}$ distribution followed by the received power in an ideal MSRC [10].

\section{MODEL VALIDATION}

In order to validate the analytical expression found in (15), we need to compare it to those obtained experimentally. The experimental setup is similar to the one presented in [7] and [5]. The setup takes place in a $13.3 \mathrm{~m}^{3} \mathrm{RC}$ equipped with a 100-step mechanical stirrer blade of $50 \mathrm{~cm}$ wide; its LUF is around $550 \mathrm{MHz}$. The relative variance is studied over the frequency range of 0.7-3 GHz. Moreover, the reverberation chamber (RC) was used in two configurations. The first one is the empty $\mathrm{RC}$; the second one consisted in loading the chamber by inserting an hybrid absorber made up of four pyramids of about 30 $\mathrm{cm}$ high, standing in the center of the RC.

We present in Fig. 1 estimated values of $\varsigma_{P}^{2}$, referred to as $\hat{\varsigma}_{P}^{2}$, obtained experimentally as a function of frequency (grey line). Note that, for the sake of 
clarity, a moving average over 5 contiguous points has been applied to lower the dispersion. We present the empty and loaded cases in the upper and lower plots, respectively. We superimposed the analytical expression (solid line) obtained in (15). The effect of the absorber can be well observed on the power variability which is much lower for the loaded case, especially at frequencies below $1 \mathrm{GHz}$.

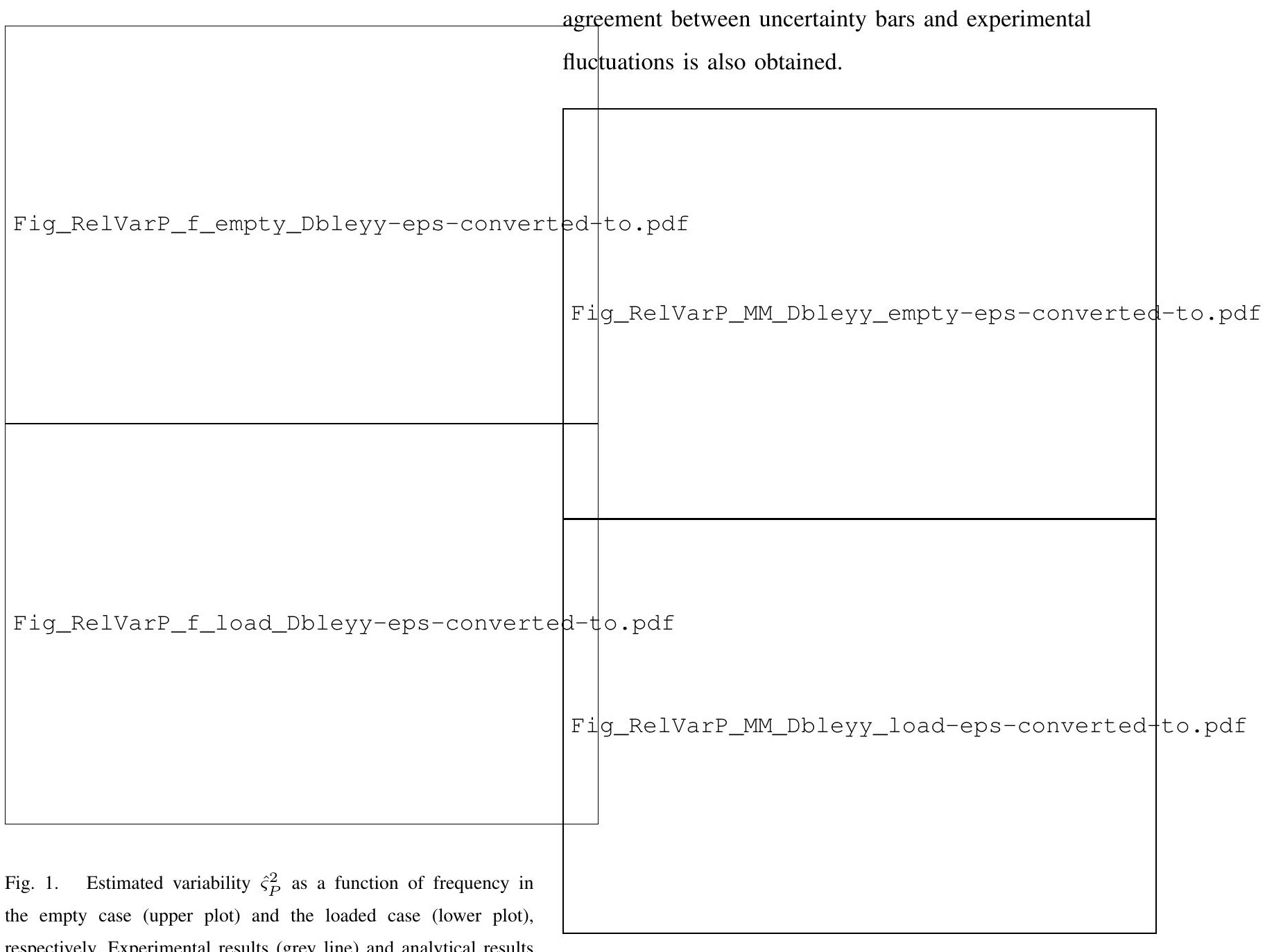
respectively. Experimental results (grey line) and analytical results (solid line) given by (15) are reported.

In order to show that the variability is indeed driven by the number $M_{M}$, we present in Fig. 2 the values of $\hat{\varsigma}_{P}^{2}$ obtained experimentally (grey curve), analytically (solid curve), and by Monte Carlo (MC) simulations (black dots) for the empty case (upper plot) and the loaded case (lower plot), respectively.

In order to estimate uncertainty bars, we need to estimate the number of independent stirrer positions. These values are given in [5] and were assumed in MC simulations that allowed us to estimate and superimpose uncertainty bars (vertical bars) related values can be read on the right $y$-axis.

We observe good agreement between analytical, numerical and experimental results. Note that satisfying agreement between uncertainty bars and experimental fluctuations is also obtained.

Fig. 2. Estimated normalized relative variance $\hat{\varsigma}_{P}^{2}$ of the electric power as a function of the average number $M_{M}$ of overlapping modes for the empty case (upper plot) and loaded case (lower plot), respectively. Experimental results (grey line), MC results (black dots) and analytical results (solid line) are reported. Horizontal and vertical bars stand for the $95 \%$ confidence intervals related to estimated values of $\hat{\varsigma}_{P}^{2}$ and $M_{M}$, respectively.

In practice, the number of independent stirrer positions depends on several parameters (degree of losses, to $95 \%$ CIs. Relative deviations from $\varsigma_{P}^{2}$ asymptotic 
stirrer shape,...). The larger this number, the more accurate the estimated average power is. From a metrology point of view, it is worth being able to predict the degree of uncertainty that one can expect in a specific configuration of the chamber.

It turns out that the results obtained previously confirm the ability of the MC code to predict the uncertainty level of the estimated power obtained experimentally, provided that the average number of overlapping modes $M_{M}$ and the number of independent stirrer positions are known.

We propose herein to use these MC simulations to estimate the degree of uncertainty of the average power for any regime of the chamber, i.e., from the undermoded to the overmoded regime. This allows us to establish a chart that could be used in practice by an MSRC engineer to check that his measurements are indeed within a given expected confidence interval (CI). Accordingly, we choose to compute CIs taken at a $95 \%$ confidence level. number of independent stirrer positions, referred to as $N_{s p}$, runs typically from less than 100 to more than a few hundred. We plot in Fig. 3 the mean value (bold line) of $\hat{\varsigma}_{P}^{2}$ and the bounds of its corresponding CI, as a function of $M_{M}$, and this, for different values of $N_{s p}$ reported on the graph; for $N_{s p}$ varying from 20 to 100 , an incremental step of 10 has been considered. Note that the case of a single measurement has been considered, i.e., no moving average on contiguous points has been performed.

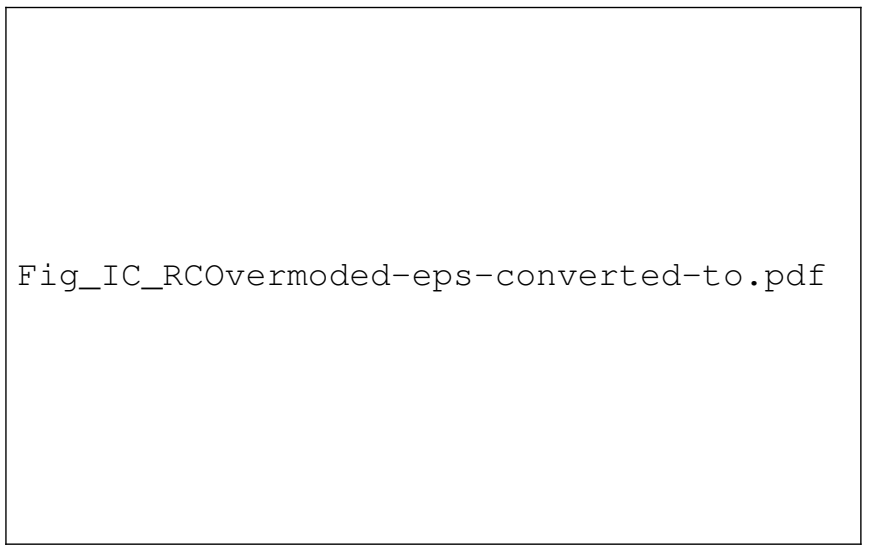

Fig. 4. Confidence intervals of $\hat{\varsigma}_{P}^{2}$ as a function of $N_{s p}$ in the overmoded case.

The case for $M_{M}=35$ can be regarded as an approximate threshold for the overmoded regime for Fig_Evol_IC_NS_v2-eps-converted-to.pdf which $\hat{\varsigma}_{P}^{2}$ is minimized. For this "best case", Fig. 4 shows the CI bounds as a function of $N_{s p}$. We can observe that the lower and upper bounds are not symmetric with respect to the asymptotic unitary mean value (dashed line), especially for low values of $N_{s p}$. A quick glance at the probability density functions ( $p d f$ )

Fig. 3. Mean value (bold line) and bounds of the confidence intervals of $\hat{\varsigma}_{P}^{2}$ as a function of $M_{M}$ for different values of $N_{s p}$. The upper and lower bounds of the $\mathrm{CI}$ are above and below the bold line, respectively.

Provided that the $\mathrm{Q}$ factor is properly assessed, the average number $M_{M}$ of overlapping modes can be regarded as a reference quantity that informs on the degree of overmodedness. It is worth stressing that the of $\hat{\varsigma}_{P}^{2}$ in the insets of Fig. 5, given for $N_{s p}=20$ and 500, respectively, shows that the approximation consisting in regarding the samples as being normally distributed cannot be stated for any value of $N_{s p}$. To assess the degree of symmetry of the $p d f$, the estimated skewness is reported. Below an inevitable arbitrary skewness threshold, the estimated variabilities could be regarded as normally distributed. 
Fig_Skewness-eps-converted-to.pdf

Fig. 5. Skewness of $\hat{\varsigma}_{P}^{2}$ for a regime approaching the overmoded case, as a function of $N_{s p}$. The histograms are shown for $N_{s p}=20$ and $N_{s p}=500$, respectively.

\section{CONCLUSION}

An analytical expression for the relative variance (or variability) of the electric power measured on a reference antenna has been derived by using a modal expansion of the electric field in a reverberation chamber. The analytic expression was found to be in good agreement with experimental results. The uncertainty of the experimental data was also found to be in good agreement with the MC simulation model.

From an application point of view, the results of this work allow one to derive confidence intervals for the variability of the power received by a reference antenna. These intervals are useful quantities for emission-test and susceptibility-test purposes.

\section{REFERENCES}

[1] Reverberation chamber test methods, International Electrotechnical Commission (IEC), Std. 61 000-4-21, 2011.

[2] M. Hoijer, "Maximum Power Available to Stress onto the Critical Component in the Equipment Under Test when Performing a Radiated Susceptibility Test in the Reverberation Chamber," IEEE Transactions on Electromagnetic Compatibility, vol. 48, no. 2, pp. 372-384, 2006.

[3] G. Orjubin, "Maximum field inside a reverberation chamber modeled by the generalized extreme value distribution," IEEE Transactions on Electromagnetic Compatibility, vol. 49, no. 1, pp. 104-113, 2007.
[4] E. Amador, H. G. Krauthauser, and P. Besnier, "A Binomial Model for Radiated Immunity Measurements," IEEE Transactions on Electromagnetic Compatibility, vol. 55, no. 4, pp. 683-691, August 2013.

[\$] F. Monsef and A. Cozza, "Average number of significant modes excited in a mode-stirred reverberation chamber," IEEE Transactions on Electromagnetic Compatibility, vol. PP(99), pp. 1-7, January 2014.

$[\phi]$ T. H. Lehman, "A Statistical Theory of Electromagnetic Fields in Complex Cavities," Interaction Notes, USAF Phillips Laboratory, vol. Note 494, May 1993.

[\}] A. Cozza, "The Role of Losses in the Definition of the Overmoded Condition for Reverberation Chambers and their Statistics," IEEE Transactions on Electromagnetic Compatibility, vol. 53, no. 2, pp. 296-307, 2011.

[8] F. Monsef, "Why a Reverberation Chamber Works at Low Modal Overlap," IEEE Transactions on Electromagnetic Compatibility, vol. 54, no. 6, pp. 1314-1317, 2012.

[9] D. Hill, "Electromagnetic Theory of Reverberation Chamber," NIST, Tech. Rep. TN 1506, December 1998.

[10] J. Kostas and B. Boverie, "Statistical Model for a Mode-Stirred Chamber," IEEE Transactions on Electromagnetic Compatibility, vol. 33, no. 4, pp. 366-370, 1991. 